\title{
Few Layer Graphene Synthesis on Transition Metal Ferrite Catalysts
}

\author{
Revathi R. Bacsa, ${ }^{1}$ Ignacio Cameán, ${ }^{2}$ Alberto Ramos, ${ }^{2}$ Ana B. Garcia, ${ }^{2}$ Victoria \\ Tishkova, ${ }^{3}$ Wolfgang S. Bacsa, ${ }^{3}$ James R. Gallagher, ${ }^{4}$ Jeffrey T. Miller, ${ }^{4}$ Hugo Navas, ${ }^{5}$ \\ Vincent Jourdain, ${ }^{5}$ Maria Girleanu, ${ }^{6}$ Ovidiu Ersen, ${ }^{6}$ Philippe Serp $^{1} *$
}

${ }^{1}$ Laboratoire de Chimie de Coordination UPR CNRS 8241, composante ENSIACET, Université de Toulouse, 4 allée Emile Monso, CS 44362, 31030 Toulouse Cedex 4, France

${ }^{2}$ Instituto Nacional del Carbón, CSIC, Francisco Pintado Fe 26, 33011-Oviedo, Spain

${ }^{3}$ Centre d'élaboration des matériaux et d'études structurales UPR CNRS 8011, 29 Rue JeanneMarvig, BP 4347, 31055 Toulouse, France

${ }^{4}$ Argonne National Laboratory, Chemical Sciences and Engineering Division, 9700 S. Cass Ave, Building 200, Argonne, IL 60429-4837, USA

${ }^{5}$ Laboratoire Charles Coulomb - UMR 5221 CNRS-UM2, Case Courrier 26 Place Eugène Bataillon, 34095 Montpellier Cedex 5, France ; CNRS, Laboratoire Charles Coulomb UMR 5221, F-34095, Montpellier, France

${ }^{6}$ IPCMS UMR 7504 CNRS-Université de Strasbourg - Département Surfaces et Interfaces (DSI), 23, rue du Lœss, BP 43, F-67034 Strasbourg, France

*Corresponding author: Fax: +33 05343235 96., E-mail address: philippe.serp@ensiacet.fr

(C) 2015. This manuscript version is made available under the Elsevier user license http://www.elsevier.com/open-access/userlicense/1.0/ 
ABSTRACT Development of cheap, green and up scalable production methods for graphene is one of the most challenging problems in its manufacture on an industrial scale. We report here a large scale substrate-free fluidized bed catalytic chemical vapour deposition (FB-CCVD) process for few layer graphene (FLG) powder production that uses a crystalline oxide catalyst of the general formula $\mathrm{A}_{\mathrm{x}} \mathrm{B}_{3-\mathrm{x}} \mathrm{O}_{4}$, wherein the FLG layer thickness and domain sizes can be varied. A and $\mathrm{B}$ can be chosen from a list of transition elements including $\mathrm{Co}, \mathrm{Fe}, \mathrm{Ni}, \mathrm{Mn}, \mathrm{Cu}$ and $\mathrm{Zn}$. The best results in terms of activity and selectivity are obtained for the $\mathrm{Co}_{\mathrm{x}} \mathrm{Fe}_{3-\mathrm{x}} \mathrm{O}_{4}$ system. We also investigated the reaction mechanism using in situ EXAFS and Raman spectroscopy, and electron tomography. Since FB-CCVD processes are already used for industrial scale production of carbon nanotubes, this process should enable the large scale production of free standing FLG in the near future.

KEYWORDS: Few layer graphene, large scale synthesis, FBCVD, Ferrite catalyst

\section{Introduction}

The wide availability of graphene and allied hybrid materials is crucial for the large scale exploitation of their unique electronic, mechanical and thermal properties.[1-5] For large volume applications such as in composites, electrochemical and energy devices, few layer graphene containing 2-10 layers (FLG) are ideal candidates, since they combine the properties of graphene

at the same time being robust and easy to handle.[6] Bottom up methods such as chemical vapour deposition (CVD) are particularly attractive in view of the fact that natural graphite, used in top-down processes, is currently a strategic material, and that its price is projected to rise in the coming years.[7] Consequently, CVD of graphene films including processes to produce and 
transfer perfect large area FLG films to any given substrate have been developed.[8-11] In contrast, very few bottom-up catalytic processes are available for the production of substratefree $\mathrm{FLG},[12,13]$ and in these processes, a precise control of thickness and crystallinity has not been demonstrated for large scale production. Indeed, the catalytic growth of carbon material from hydrocarbons is an well known but extremely complex reaction, the exact mechanism of which being still under debate. Initially, this reaction was studied due to the fact that carbon deposition was a serious cause of catalyst deactivation [14] or reactor degradation in some high temperature reactions such as steam reforming, methanation, and other important catalytic processes involving iron, cobalt and nickel catalysts.[15-17] Basically, three types of deposits have been regularly reported: i) flocculent amorphous carbon, ii) crystalline platelets or flakes of graphite, and iii) fibrous, polycrystalline carbon.[18] Three-dimensionally ordered graphitic flakes with 200-500 $\mathrm{nm}$ thickness have been successfully produced in the past on $\mathrm{Fe}$, Co, Ni or Pt foils or films from methane or acetylene.[16, 17, 19] The use of Fe nanoparticles, instead of film, as catalyst and $\mathrm{CO}$ as carbon source allows the selective formation of fibrous carbon [20, 21]. Thus a breakthrough in the form of a selective, large scale and inexpensive process for the production of free standing crystalline FLG that allows a high control of thickness and defect density is highly desired before FLG commercialization.

Here, we report a fluidized-bed catalytic CVD (FB-CCVD)[22] process to produce FLG in high yield and we have investigated the mechanism of its formation. The process consists of the catalytic decomposition of a carbon source such as ethylene or ethane over Co-Fe alloyed catalyst particles, which are produced in situ during the reduction of a nanocrystalline spinel material of the family $\mathrm{A}_{\mathrm{x}} \mathrm{B}_{3-\mathrm{x}} \mathrm{O}_{4}$. While we have checked that several members from the family of spinels can be successfully used for the selective formation of FLG (A = Co, Ni, Mn, $\mathrm{Cu}, \mathrm{Zn}$ and 
$\mathrm{B}=\mathrm{Fe}$ ), best results in terms of activity and selectivity have been obtained so far for $\mathrm{A}=\mathrm{Co}$ and $\mathrm{B}=\mathrm{Fe}$. The yield and thickness are determined by a variety of parameters including i) catalyst composition ii) crystallite size of the spinel and iii) the reaction conditions of the catalytic CVD process. In the following sections, we show that the spinel oxides form a unique system for FLG growth in that: i) the in situ formation of Fe-Co alloy induces metal dusting to nucleate carbon, and ii) by varying the $\mathrm{Fe} / \mathrm{Co}$ ratio, the rate of carbon deposition can be fine-tuned to achieve thickness control. We have also reported that a catalyst composition containing a mixture of the $\mathrm{CoFe}_{2} \mathrm{O}_{4}$ and a standard multiwall carbon nanotube (MWCNT) catalyst[23] leads to MWCNTFLG hybrids with composition control in a single step.[24]

\section{Experimental}

\subsection{FLG synthesis}

In a typical experiment, $25 \mathrm{mg}-1 \mathrm{~g}$ of the catalyst was inserted into a horizontal fixed bed or a vertical fluidized bed reactor and reduced under $\operatorname{Ar}\left(\mathrm{N}_{2}\right) / \mathrm{H}_{2}$ (3:1) flow for $1 \mathrm{~h}$ to temperatures in the range $650-700^{\circ} \mathrm{C}$, after which $0.03-1 \mathrm{~L} / \mathrm{min}$ of ethylene gas is introduced for a varying period of time ranging from $9 \mathrm{~s}-1 \mathrm{~h}$. The reaction products are cooled under $\mathrm{Ar} / \mathrm{N}_{2}$. The yield of the reaction was calculated by the parameter $\xi=\mathrm{g}_{\mathrm{FLG}} / \mathrm{g}_{\text {catalyst }}$. The reduced catalyst with the carbon deposit is dropped into $35 \% \mathrm{HCl}$ at $20^{\circ} \mathrm{C}$ to dissolve the catalyst. The carbon powder is washed with water and dried at $120^{\circ} \mathrm{C}$ in air. The carbon content in the washed sample was measured using thermogravimetric analysis (TGA) and the oxygen content was estimated from XPS.

\subsection{Heat treatment of FLG particles}

The heat treatment of the FLG samples was carried out in the temperature interval of 2200-2800 ${ }^{\circ} \mathrm{C}$ in a graphite electrical furnace for $1 \mathrm{~h}$ under Ar flow. This treatment was done to study the 
effect of high temperature annealing on the purity and crystallite size growth of the samples. [21] The heating rates were $50{ }^{\circ} \mathrm{C} \mathrm{min}{ }^{-1}, 100{ }^{\circ} \mathrm{C} \mathrm{min}{ }^{-1}, 25{ }^{\circ} \mathrm{C} \min -1$ and $10{ }^{\circ} \mathrm{C} \mathrm{min}{ }^{-1}$ in the temperature intervals of room to $700{ }^{\circ} \mathrm{C}, 700-1000^{\circ} \mathrm{C}, 1000-2000^{\circ} \mathrm{C}$ and $2000-2800^{\circ} \mathrm{C}$, respectively. The resulting graphitic materials were denoted by including a suffix with the treatment temperature in the GP designation, such as GP/2400 or GP1/2400.

\subsection{Characterization techniques}

Scanning electron microscopy images were taken on a Field Effect Gun SEM JEOL 6700F microscope. Low resolution TEM images were done on a JEOL 1011 instrument and a FEG Schottky JEOL 2010F analytical electron microscope, equipped with a field-emission gun was used for high-resolution transmission electron microscopy (HRTEM) investigations. Chemical analysis of the nanoparticles was carried out in STEM configuration (spot mode, probe diameter $=1 \mathrm{~nm}$ ) using a Bruker EDAX spectrometer (provided with a Silicon Drift Detector with a resolution $127 \mathrm{eV}$ ). The X-ray diffractograms were recorded in a Bruker D8 powder diffractometer equipped with a göbel mirror in the incident beam and a parallel-slits analyzer in the diffracted beam. The mean crystallite sizes, $\mathrm{L}_{\mathrm{c}}$ and $\mathrm{L}_{\mathrm{a}}$, were calculated from the (002) and (110) peaks, respectively, using the Scherrer formula, with values of $K=0.9$ for $L_{c}$ and 1.84 for $\left.\mathrm{L}_{\mathrm{a}}\right]$.

Raman spectra were obtained in a Raman micro spectrometer HR 800 Jobin Yvon Horiba using the green line of an argon laser $(\lambda=532 \mathrm{~nm})$ as an excitation source. The intensity (integrated area) I and width (FWHM) W of the bands were measured using a mixed Gaussian-Lorentzian curve-fitting procedure. 
X-ray absorption measurements were acquired at the Fe $(7.112 \mathrm{keV})$ and Co K-edges (7.709 $\mathrm{keV}$ ) on the bending magnet beam line of the Materials Research Collaborative Access Team (MRCAT) at the Advanced Photon Source, Argonne National Laboratory. The data was collected in transmission step-scan mode. Photon energies were selected using a water-cooled, double-crystal $\mathrm{Si}(111)$ monochromator, which was detuned by approximately $50 \%$ to reduce harmonic reflections. The ionization chambers were optimized for the maximum current with linear response ( $\sim 1010$ photons detected/sec) with $10 \%$ absorption in the incident ion chamber and $70 \%$ absorption in the transmission X-ray detector. A Fe or Co foil spectrum was acquired simultaneously with each sample measurement for energy calibration.

Standard data reduction techniques were employed to fit the data using the WINXAS 3.1 software program. The normalized, energy-calibrated absorption spectra were obtained using standard methods. The pre-edge edge energy of oxidized samples was determined by the peak energy position of the pre-edge. The edge energy was determined from the maximum of the first peak by taking the first derivative of the XANES spectrum. Experimental phase shift and backscattering amplitudes were measured using standard compounds of known structure, Co (12 Co-Co at $2.51 \AA)$ and Fe foil (8 Fe-Fe at $2.48 \AA$ ). The EXAFS parameters were obtained by a least square fit in R-space of the k2-weighted Fourier Transform (FT) data. The Debye-Waller factor $\left(\Delta \sigma^{2}\right)$ is temperature dependent and reduced $\mathrm{Fe}$ and $\mathrm{Co}$ were measured at $650{ }^{\circ} \mathrm{C}$ and again after cooling to RT. The $\Delta \sigma^{2}$ fit parameter for data at $650^{\circ} \mathrm{C}$ was determined assuming the coordination number was unchanged at lower temperature. For other samples, $\Delta \sigma^{2}$ was held constant for high temperature fits.

TPR spectrum was obtained with a fully automated AMI-200 Catalyst Characterization Instrument (Altamira Instruments), equipped with a thermal conductivity detector (TCD) and a 
quadrupole mass spectrometer (Dymaxion $200 \mathrm{amu}$, Ametek). The sample was placed in a Ushaped quartz tube located inside an electrical furnace and heated at $5 \mathrm{~K} / \mathrm{min}$ to $973 \mathrm{~K}$ under a 5 vol.\% $\mathrm{H}_{2}$ flow diluted with $\mathrm{He}$ (total flow rate of $30 \mathrm{~cm}_{3} / \mathrm{min}, \mathrm{STP}$ ). The $\mathrm{H}_{2}$ consumption was followed by both TCD and mass spectrometry.

For in situ Raman measurements the stage was calcined under air (between 650 and $800^{\circ} \mathrm{C}$ depending on experiments) before catalyst treatment. $\mathrm{CoFe}_{2} \mathrm{O}_{4}$ catalyst was deposited as individual grains on a $\mathrm{SiO}_{2}-\mathrm{Al}_{2} \mathrm{O}_{3}$ ceramic cloth (provided with the Linkam CCR 1000 stage). The temperature was ramped under $\mathrm{Ar} / \mathrm{H}_{2}$ by steps up to $650^{\circ} \mathrm{C}$. In situ Raman monitoring was performed in confocal micro-Raman mode with a laser excitation of $532 \mathrm{~nm}$ at a laser power of $0.8 \mathrm{~mW}$ under the objective (x50).

The tomographic 3D analysis in conventional bright field TEM, was performed using a JEOL 2100F TEM/STEM microscope, operating at $200 \mathrm{kV}$ and equipped with a probe corrector and a GIF Tridiem energy filter. Before observation, the powdered material was suspended in ethanol by sonication and several droplets were deposited onto a cooper grid covered by a carbon holey membrane. The acquisitions of the tilt series were performed using high tilt sample holder and acquisition software from GATAN Company. The images tilt series was acquired with angles spanning from +60 to -70 degree, with projections taken every $2^{\circ}$ according to the Saxton scheme.

\subsection{Catalyst treatments}

Catalysts were measured using a continuous-flow reactor, which consisted of a quartz tube (1-in.

OD, 10-in. length) sealed with Kapton windows by two Ultra-Torr fittings. Ball valves were welded to each Ultra-Torr fitting and served as the gas inlet and outlet. An Omega K-type 
thermocouple was placed against the catalyst sample holder to monitor temperature. Catalyst samples were pressed into a cylindrical sample holder consisting of six wells, forming a selfsupporting wafer. Samples were diluted with the carbon provided to achieve ca $0.35-0.40 \mathrm{mg}$ metal in the beam, which gives an absorbance $\left(\mu_{\mathrm{x}}\right)$ of approximately 1.0. Traces of oxygen and moisture in the He were removed using a Matheson PUR-Gas Triple Purifier Cartridge.

Catalysts were first measured under air at RT. Samples were then reduced in $3.5 \% \mathrm{H}_{2} / \mathrm{He}$ at 650 ${ }^{\circ} \mathrm{C}$ before being cooled to RT for measurement. The same samples were then heated under $3.5 \%$ $\mathrm{H}_{2} / \mathrm{He}$ for measurement at $650^{\circ} \mathrm{C}$ on the beamline. After measurement, whilst still at $650{ }^{\circ} \mathrm{C}$ the gases were switched to a mixture of pure $\mathrm{H}_{2}(30 \mathrm{ml} / \mathrm{min})$ and $4 \% \mathrm{C}_{2} \mathrm{H}_{4} / \mathrm{He}(90 \mathrm{ml} / \mathrm{min})$ and the samples treated for $30 \mathrm{~min}$. Measurements were taken at $650{ }^{\circ} \mathrm{C}$ in $\mathrm{C}_{2} \mathrm{H}_{4} / \mathrm{H}_{2}$. Finally, the reactor was purged with $3.5 \% \mathrm{H}_{2} / \mathrm{He}$ and cooled to RT where the final measurements were taken.

\section{Results and discussion}

The oxide spinel catalysts $\mathrm{Co}_{\mathrm{x}} \mathrm{Fe}_{3-\mathrm{x}} \mathrm{O}_{4}$ reported in this work have been synthesized by the decomposition of the corresponding citrates.[25] The crystal structure, crystallite size and composition of the catalysts were evaluated from X-ray diffractograms, wherefrom lattice parameters were calculated (Figure S.1). Crystallite sizes were modified both by heat treatment in air for a given composition (Figure S.1b), and by varying the $\mathrm{Co} / \mathrm{Fe}$ ratio (Figure S.2). $\mathrm{Co}_{\mathrm{x}} \mathrm{Fe}_{3}$ ${ }_{\mathrm{x}} \mathrm{O}_{4}$ with crystallite sizes ranging from $10-100 \mathrm{~nm}$ and $x$ ranging from $0-3$ were prepared and tested for FLG synthesis. Figure 1A shows the variation of FLG yield $\xi\left(\mathrm{g}_{\mathrm{FLG}} / \mathrm{g}_{\text {catalyst }}\right)$ with time for FLG growth from ethylene at $650^{\circ} \mathrm{C}$ on the $\mathrm{CoFe}_{2} \mathrm{O}_{4}$ catalyst (mean crystallite size $14 \mathrm{~nm}$ ). $\xi$ increases rapidly with time during the first 20 min of reaction. As produced FLGs were purified by $\mathrm{HCl}$ treatment at room temperature. The chemical purity as measured by TGA was 
found to be $98.5 \%$ (time of synthesis $=60 \mathrm{~min}$ ) (Figure S.3). XPS analysis showed that oxygen content in the purified samples was less than 4 at. \% (Figure S.4). Figures 1B and 1C show the SEM and TEM images of the corresponding FLG particles. The powder consists agglomerates of 80 to $100 \mu \mathrm{m}$ made up of flakes of uniform diameter $(0.2-0.25 \mu \mathrm{m})$ tending to curl up at the edges. TEM of these flakes (Fig. 1C) show stacked crystalline layers of graphene. Domain sizes as measured from TEM images vary from $10-30 \mathrm{~nm}$ and are much smaller than the flake sizes. A histogram of thickness was plotted by observing a number of flakes and tilting the sample (Fig. 1C). It is observed that after 9s of reaction, the number of layers is in the 1-5 range. AFM images of dilute dispersions in THF deposited on a silicon substrate have confirmed this (S.5). 
A

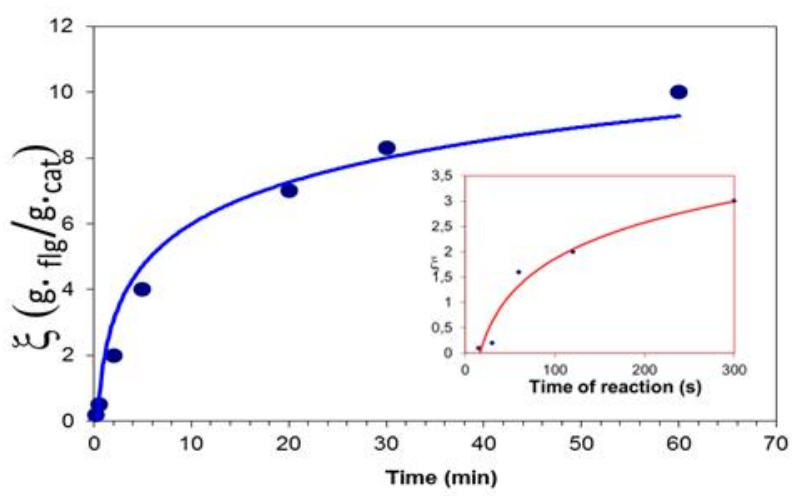

C

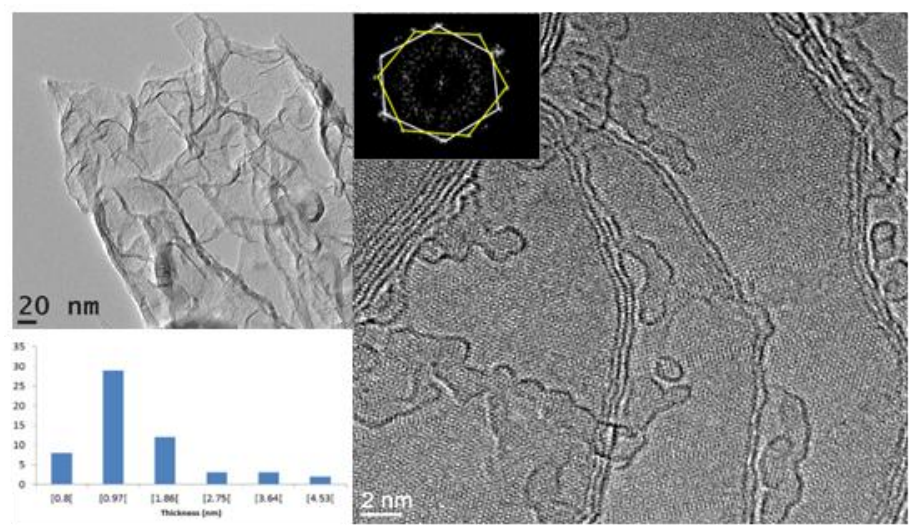

$\mathrm{E}$

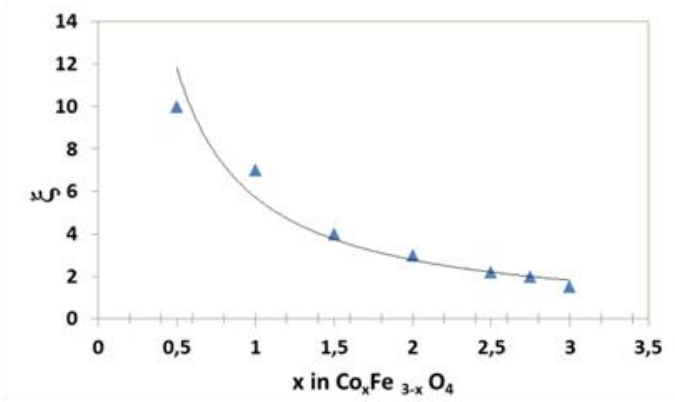

$\mathrm{F}$
B

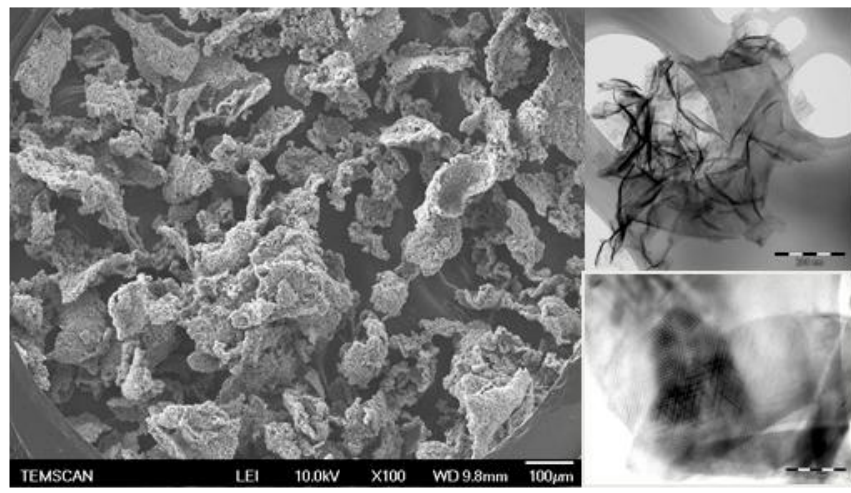

D
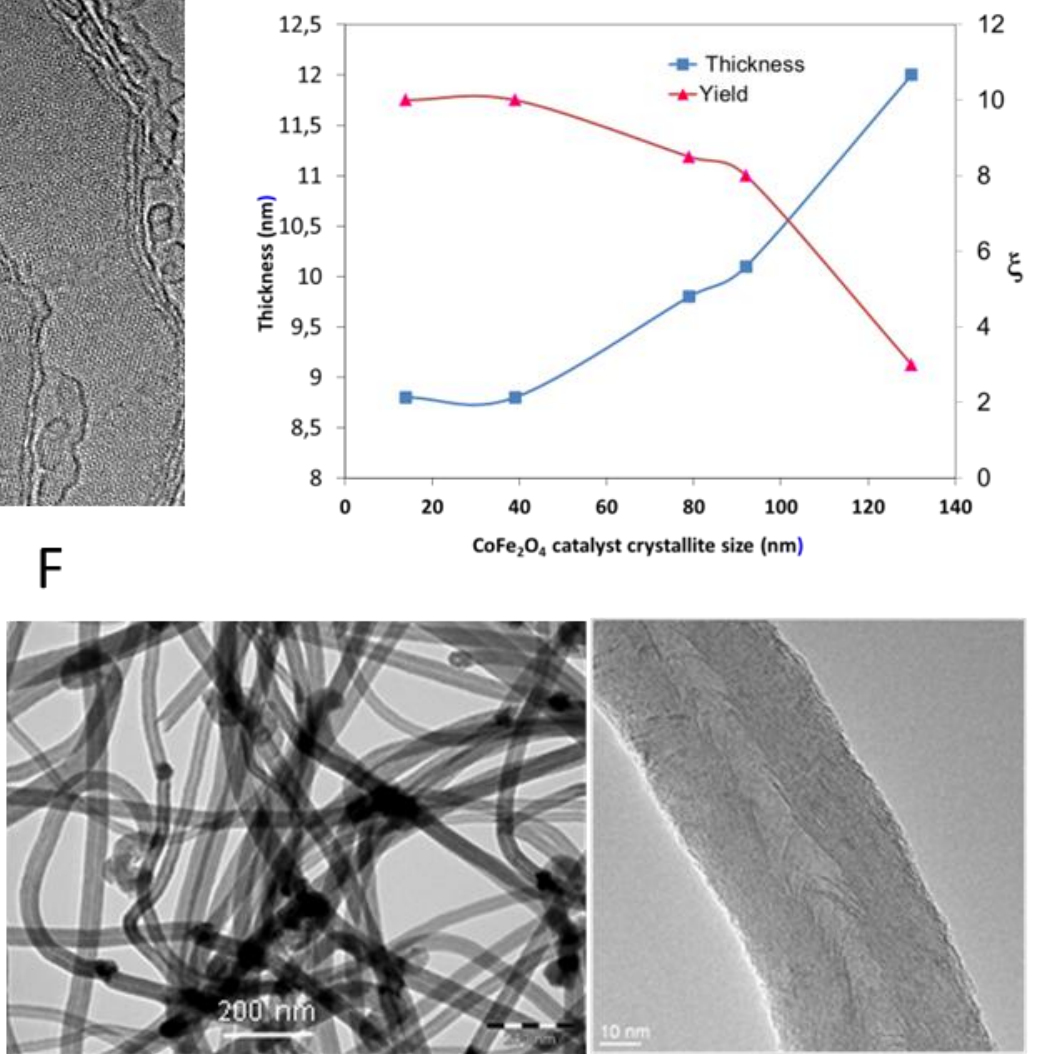

Figure 1A CVD reaction yield with time of synthesis. Inset shows average FLG thickness (as estimated from XRD) as a function of time is shown.1B SEM image of FLG flakes. (Right) TEM images (scale bar $=300 \mathrm{~nm}$ (up) and $100 \mathrm{~nm}$ (down)) showing a single flake and several cristalline flakes superposed on each other showing a Moire pattern.1C TEM image of FLG flakes tilted to observe the thickness with a histogram of thickness for 9s of reaction time. On the right is shown the surface of FLG after heat treatment at $2800^{\circ} \mathrm{C}$ and the Fourier transformed image indicating rotated graphene planes. 1D Variation of $\xi$ and average thickness with crysallite sizes for $\mathrm{CoFe}_{2} \mathrm{O}_{4}$ catalyst. 1E: $\xi$ as a function of $\mathrm{x}(1 \mathrm{~h}$ reaction). $1 \mathrm{~F}$ (left) Carbon nanofibers 
produced with $\mathrm{CoFe}_{2} \mathrm{O}_{4}$ catalyst at $450^{\circ} \mathrm{C}$ (scale bar $=200 \mathrm{~nm}$ ) and (right) TEM image of a single fiber.

An estimate of the average thickness can also be determined from the full width at half maximum of the (002) reflection in the X-ray powder diffractograms using the Scherrer equation. These increase with reaction time and reach a plateau at $7.5 \mathrm{~nm}$ (Figure 1A, inset). For the $\mathrm{CoFe}_{2} \mathrm{O}_{4}$ catalyst, the effect of oxide crystallite size on $\xi$ and thickness of the FLG is shown in Figure 1D. It is observed that increasing the crystallite size of the catalyst decreases $\xi$ and leads to thicker FLG. All the spinels of the general formula $\mathrm{Co}_{\mathrm{x}} \mathrm{Fe}_{3-\mathrm{x}} \mathrm{O}_{4}$ functioned as catalysts for the FLG production. The oxide crystallite sizes varied only slowly with change in $x$ (10-15 $\mathrm{nm}$ ) with the exception of $\mathrm{Co}_{3} \mathrm{O}_{4}(26 \mathrm{~nm})$ (Figure S.2b). However, the FLG yield $\xi$ decreased with increase in $x$ values (Figure 1E), with $\mathrm{Co}_{3} \mathrm{O}_{4}$ showing very little carbon deposition $\left(\mathrm{Fe}_{3} \mathrm{O}_{4}\right.$ is not considered since it did not form FLG but gave rise to filamentous carbon). Corresponding TEM images showed that the average diameter of the flakes increased from 200 to $500 \mathrm{~nm}$ upon changing the composition from $\mathrm{CoFe}_{2} \mathrm{O}_{4}$ to $\mathrm{Co}_{2.75} \mathrm{Fe}_{0.25} \mathrm{O}_{4}$. Raman spectra of FLG produced therefrom, showed a slight decrease in the ratio of the intensities of the D band to the G band $\left(\mathrm{I}_{\mathrm{D}} / \mathrm{I}_{\mathrm{G}}\right)$ from 1.6 to 1.2 (Figure $\mathrm{S} .6$ ) that could be related to the increase in the flake size.[26] Decreasing $x$ increases the yield but decreases selectivity with the formation of filamentous carbon at values $x<0.5$.

Fourier transform of TEM images of FLG (Figure 1C) have shown that these consist of more than one graphene layer rotated with respect to each other with random rotational angles, as has been reported for multilayer graphene films produced on $\mathrm{Cu}$ substrates.[27] The random stacking has also been probed using Raman analysis of purified samples as well as FLG annealed between 
$2200^{\circ} \mathrm{C}$ and $2800^{\circ} \mathrm{C}$ in argon atmosphere. A Raman analysis of the samples is presented in Figure 2 and the Raman parameters are given in Table 1.

\begin{tabular}{|l|lll|l|l|l|l|l|l|l|l|}
\hline & \multicolumn{3}{|c|}{ XRD } & \multicolumn{7}{c|}{ RAMAN } \\
\hline Materials & $\mathrm{d}_{002}(\mathrm{~nm})$ & $\mathrm{L}_{\mathrm{c}}(\mathrm{nm})$ & $\mathrm{L}_{\mathrm{a}}(\mathrm{nm})$ & $\begin{array}{l}\mathrm{W}_{\mathrm{D}} \\
\left(\mathrm{cm}^{-1}\right)\end{array}$ & $\begin{array}{l}\mathrm{W}_{\mathrm{G}} \\
\left(\mathrm{cm}^{-1}\right)\end{array}$ & $\begin{array}{l}\mathrm{I}_{\mathrm{D}} / \mathrm{I}_{\mathrm{G}} \\
(\%)\end{array}$ & $\begin{array}{l}\mathrm{I}_{\mathrm{D}} / \mathrm{I}_{\mathrm{G}} \\
(\%)\end{array}$ & $\begin{array}{l}\mathrm{W}_{\mathrm{G}^{\prime}} \\
\left(\mathrm{cm}^{-1}\right)\end{array}$ & $\mathrm{I}_{\mathrm{G}^{\prime} 3 \mathrm{DA}}$ & $\mathrm{I}_{\mathrm{G}^{\prime} 2 \mathrm{D}}$ & $\mathrm{I}_{\mathrm{G}^{\prime} 3 \mathrm{DB}}$ \\
\hline FLG & 0.3400 & 11.1 & 20.8 & 42.7 & 31.3 & 73.1 & 7.8 & 60.4 & 0.29 & 0.53 & 0.18 \\
FLG/2200 & 0.3403 & 13.3 & 29.9 & 38.7 & 23.5 & 25.0 & 3.4 & 49.6 & 0.34 & 0.53 & 0.13 \\
FLG/2400 & 0.3401 & 14.0 & 32.5 & 39.2 & 23.1 & 23.0 & 3.5 & 50.2 & 0.36 & 0.56 & 0.08 \\
FLG/2600 & 0.3401 & 14.5 & 31.7 & 39.0 & 21.9 & 19.0 & 3.0 & 49.0 & 0.40 & 0.40 & 0.20 \\
FLG/2800 & 0.3397 & 15.3 & 34.7 & 39.0 & 21.4 & 17.8 & 3.0 & 48.7 & 0.40 & 0.48 & 0.12 \\
\hline
\end{tabular}

Table 1 Crystalline parameters from XRD and Raman for purified FLG before and after heat treatment.

The spectra are highly uniform over the entire flake. The narrow $\mathrm{G}$ band at 9s shows that a well crystallized $s p^{2}$ carbon network is present. 

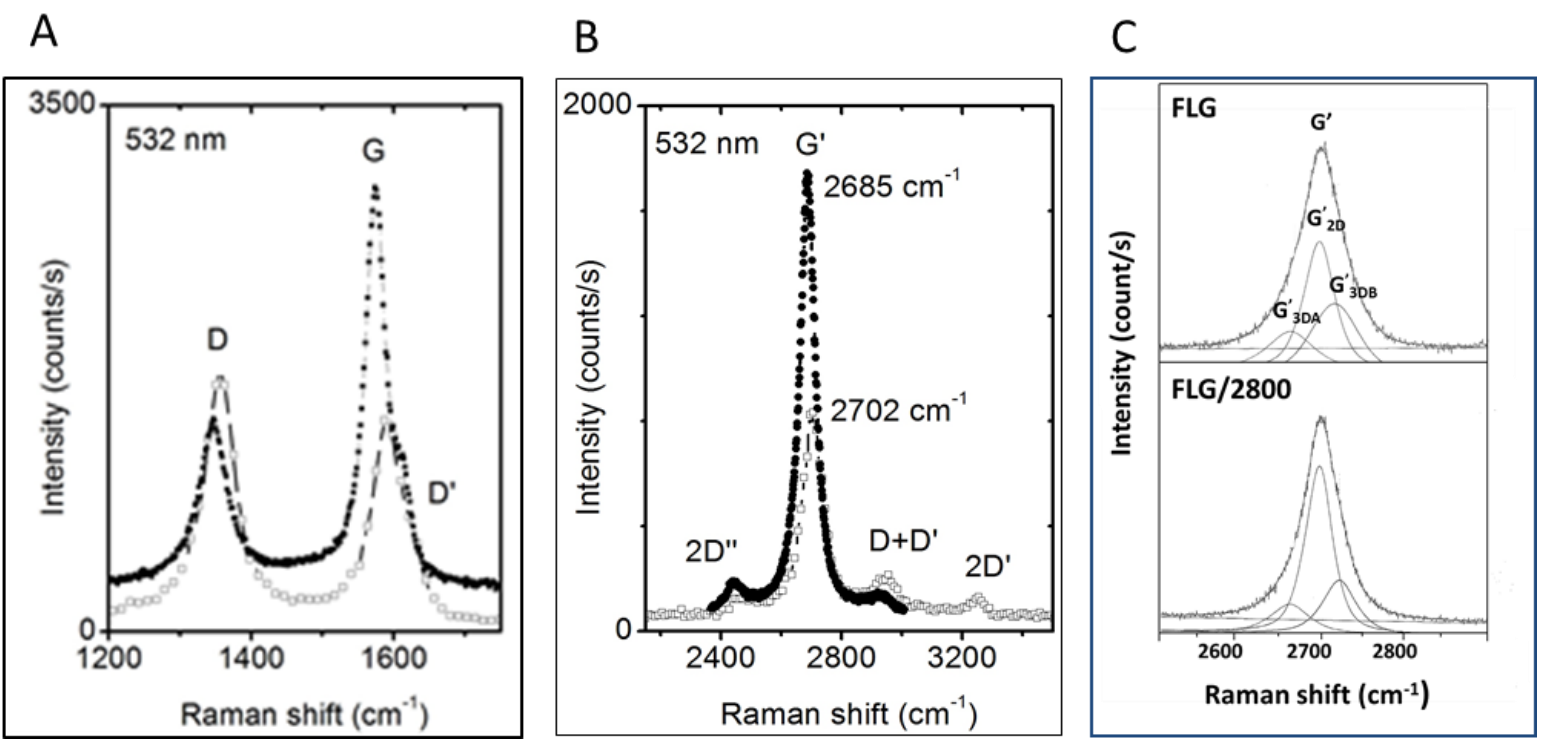

Figure 2. Raman spectrum (laser excitation $532 \mathrm{~nm}$ ) in low (A) and high (B) energy range of FLGs at two different process times (dark line: $9 \mathrm{~s}$ and light line: $20 \mathrm{~min}$ ). (C) The three peak deconvolution of the G'-band of the FLG and heat-treated FLG materials

The D and D' bands are relatively high, that could be the result of defects at edges of smaller flakes, the small area of flakes giving rise to a large number of edges. An intense G' band is one of the indications for the presence of graphene.[28] Figure 2B shows a symmetric G' band with up to three times the intensity of the G band as for graphene, and the width of this band is similar to single layer graphene indicating random layer stacking. The less intense band corresponding to the longer process time in Fig. 2B is upshifted and the two band positions are compatible with graphene $\left(2685 \mathrm{~cm}^{-1}\right)$ and turbostratic graphite $\left(2702 \mathrm{~cm}^{-1}\right)$. Both $\mathrm{G}^{\prime}$ band positions are distinctly different from graphite $\left(2721 \mathrm{~cm}^{-1}\right)$. The side bands are assigned to second order defect induced bands.[26] This result indicates a transition from single layer graphene-like to turbostratic graphite-like behaviour with increased processing time or increased thickness (Figure 1A). 
We have studied the effect of argon annealing on the domain sizes and stacking using Raman spectroscopy and X-ray diffraction (Figures. S.7-S.9). The results are summarized in Table 1. The widths of all the XRD reflections decrease with increasing annealing temperature (2200$2800{ }^{\circ} \mathrm{C}$ ) showing an increase in structural order. The (002) reflection shifts to larger angles (in particular for $2800{ }^{\circ} \mathrm{C}$ ) showing a decrease in $\mathrm{d}_{002}$ and a larger mean crystallites sizes that is especially pronounced along the $a$-axis (from $20 \mathrm{~nm}$ to $35 \mathrm{~nm}$ ). However, the absence of the (112) reflection in the XRD profiles even at $2800{ }^{\circ} \mathrm{C}$, indicates a very low development of the three-dimensional stacking. Thus it appears that a preferential development of bi-dimensional structure occurs, as confirmed by the higher growth of the crystallite size along the basal plane, $\mathrm{L}_{\mathrm{a}}$. The small peak due to residual catalyst particles at $2 \theta=44.6^{\circ}$ present in purified FLG is absent in heat treated samples probably due to the sublimation of the FeCo alloy.

The Raman parameters from the first-order spectra are also reported in Table 1. The defect induced D band narrows $\left(\mathrm{W}_{\mathrm{D}}\right)$ and gets less intense with annealing at $2200{ }^{\circ} \mathrm{C}$, which is in accordance with increased in $\mathrm{L}_{\mathrm{a}}$. But no large increase is observed for higher annealing temperatures. Similarly, the width of the $\mathrm{G}$ band $\left(\mathrm{W}_{\mathrm{G}}\right)$ decreases from $\sim 31 \mathrm{~cm}^{-1}$ to $\sim 21 \mathrm{~cm}^{-1}$ after annealing at $2800{ }^{\circ} \mathrm{C}$. The $\mathrm{I}_{\mathrm{D}} / \mathrm{I}_{\mathrm{G}}$ and $\mathrm{I}_{\mathrm{D}} / \mathrm{I}_{\mathrm{G}}$ values show a corresponding decrease. The $\mathrm{D}$ band has been found to be more intense at edges of graphene. In our case, on annealing, the D band is reduced by a factor of 4 , whereas the D' band is relatively less affected (reduction $<3$ ). This indicates that annealing reduces mainly the edge defects as is expected for crystal growth through coalescence. The $\mathrm{W}_{\mathrm{G}}$, of FLG decreases when annealing at $2200{ }^{\circ} \mathrm{C}$, but shows little change thereafter up to $2800^{\circ} \mathrm{C}$. The number of graphene layers is known to influence the $\mathrm{G}$ band shape. The G' band can best be fitted by three Lorentz peaks. Figure $2 \mathrm{C}$ shows the deconvolution of the G' band. Three peaks are observed, one at the same frequency of the 
original $\mathrm{G}^{\prime}$ at around $2698 \mathrm{~cm}^{-1}$ (denoted $\mathrm{G}_{2 \mathrm{D}}$ due to its association with a two dimensional character associated with a poor stacking order along the $c$ axis), and two others at around 2687 $\mathrm{cm}^{-1}$ and $2727 \mathrm{~cm}^{-1}$ denoted $\mathrm{G}_{3 \mathrm{DA}}$ and $\mathrm{G}_{3 \mathrm{DB}}$, which are associated with the 3 dimensional configuration of graphite.[29, 30] After annealing, while $\mathrm{I}_{\mathrm{G}}{ }^{3 \mathrm{D}}$ increases and $\mathrm{I}_{\mathrm{G}}{ }^{2} \mathrm{D}$ decreases

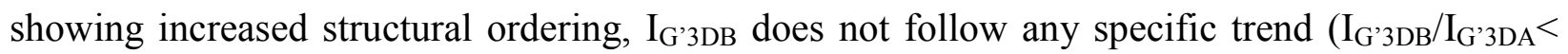
1). Hence, the high temperature treatment of FLG improves the graphitic structural order, particularly the bi-dimensional crystalline order[31] but has little effect on the stacking order.

X-ray diffraction of unpurified FLG at 9s reaction (Figure S.10) indicated that the spinel catalyst undergoes complete reduction during the CVD reaction to produce Fe-Co alloys with the $b c c$ structure. A low intensity peak that could be attributed to cobalt ferrite spinel was observed, probably due to the alloy nanoparticle oxidation upon air exposure. The reaction selectivity and hence the nature of the active phase depends critically on the temperature of synthesis. Carbon nanofibers (CNFs) with diameters ranging from 30-60 nm (Figure 1F) were formed selectively at $450{ }^{\circ} \mathrm{C}(\xi=3.5)$. $\xi$ increased to 4 at $500{ }^{\circ} \mathrm{C}$ and decreased to 2.7 at $550{ }^{\circ} \mathrm{C}$. The selective production of CNFs by CVD using a support-free catalyst has not been reported yet. FLG was selectively formed in the temperature window of $620-720^{\circ} \mathrm{C}$, and higher temperatures lowered the selectivity of the reaction with the simultaneous formation of MWCNT. The ferrite reduction and the reaction of the active phase with ethylene were studied using in situ XANES, EXAFS and Raman. TEM observations were performed ex situ.

To understand the nature of the unsupported spinel catalysts under reaction conditions, the oxidation state and coordination environment were determined by in situ XANES and EXAFS 
(Figures S.10- S.14) after reaction in $\mathrm{H}_{2}$ (S.15-S.18) and in $\mathrm{H}_{2}+\mathrm{C}_{2} \mathrm{H}_{4}$ (S.19-S.21) for three materials; monometallic $\mathrm{Co}_{3} \mathrm{O}_{4}, \mathrm{Fe}_{3} \mathrm{O}_{4}$ and $\mathrm{CoFe}_{2} \mathrm{O}_{4}$. The XANES and EXAFS of the FeCo catalyst before reduction (Figures S.11-S.14) were consistent with formation of a mixed metal oxide, while after reduction an alloy phase with $b c c$ structure was formed, consistent with the ex situ XRD data. Fitted EXAFS parameters are detailed in Table 2 and show that the data measured at $650{ }^{\circ} \mathrm{C}$ are consistent with the RT data, suggesting that the alloy is stable and fully reduced at both room temperature and $650{ }^{\circ} \mathrm{C}$. Treatment with $\mathrm{H}_{2}+\mathrm{C}_{2} \mathrm{H}_{4}$ caused a small change to the XANES spectrum of the pure Fe nanoparticles indicating that $\mathrm{H}_{2}+\mathrm{C}_{2} \mathrm{H}_{4}$ modified the structure (Figure S.19). The shape of the XANES was similar to metallic Fe, but with slightly less pronounced peaks. XANES spectra taken at $650{ }^{\circ} \mathrm{C}$ were very similar to those taken at $r t$. The EXAFS also changed after $\mathrm{H}_{2}+\mathrm{C}_{2} \mathrm{H}_{4}$ treatment (Fig. 3B) and the Fe-Fe coordination number decreased from 6.3 to 4.2 indicating that the size of the Fe nanoparticles decreases significantly under reaction conditions due to metal dusting.[32] The Fe-Fe bond distance was much shorter than in bulk metallic Fe (2.33 vs. $2.48 \AA$, respectively) consistent with very small $(c a<3 \mathrm{~nm})$ nanoparticles. This bond distance does not fit the Fe-Fe bond distance in iron carbides and there was no evidence of a Fe-C bond. Thus, under reaction conditions Fe nanoparticles decrease in size without formation of iron carbides. The XANES and EXAFS results at $650{ }^{\circ} \mathrm{C}$ are similar to those at RT with the only differences being attributable to the changes in thermal disorder with temperature, suggesting that the phase that was formed was stable at RT. The pure Co nanoparticles did not change after treatment with $\mathrm{H}_{2}+\mathrm{C}_{2} \mathrm{H}_{4}$ (no dusting observed) with the coordination number being almost identical to that after reduction (10.8 increasing to 11.1) and no evidence for carbide formation. 


\begin{tabular}{|c|c|c|c|c|c|c|c|}
\hline Sample & Treatment & Measurement & $\begin{array}{c}\text { Scattering } \\
\text { path }\end{array}$ & $N$ & $\begin{array}{c}R \\
(\AA)\end{array}$ & $\begin{array}{c}\Delta \sigma^{2} \\
x 10\end{array}$ & $\begin{array}{c}E_{0} \\
(\mathrm{eV})\end{array}$ \\
\hline Fe foil & Standard & Air, RT & $\mathrm{Fe}-\mathrm{Fe}$ & 8 & 2.48 & $\mathrm{~N} / \mathrm{A}$ & N/A \\
\hline Co foil & Standard & Air, RT & $\mathrm{Co}-\mathrm{Co}$ & 12 & 2.51 & $\mathrm{~N} / \mathrm{A}$ & N/A \\
\hline $\mathrm{Fe}_{3} \mathrm{O}_{4}$ & $\mathrm{H}_{2}, 650^{\circ} \mathrm{C}$ & $\mathrm{H}_{2}, \mathrm{RT}$ & $\mathrm{Fe}-\mathrm{Fe}$ & 6.3 & 2.49 & 1.0 & 3.5 \\
\hline $\mathrm{Fe}_{3} \mathrm{O}_{4}$ & $\mathrm{H}_{2}, 650^{\circ} \mathrm{C}$ & $\mathrm{H}_{2}, 650^{\circ} \mathrm{C}$ & $\mathrm{Fe}-\mathrm{Fe}$ & 6.3 & 2.47 & 13.0 & -0.4 \\
\hline $\mathrm{Fe}_{3} \mathrm{O}_{4}$ & $\mathrm{H}_{2}+\mathrm{C}_{2} \mathrm{H}_{4}, 650^{\circ} \mathrm{C}$ & $\mathrm{H}_{2}, \mathrm{RT}$ & $\mathrm{Fe}-\mathrm{Fe}$ & 4.2 & 2.33 & 8.0 & 0.1 \\
\hline $\mathrm{Fe}_{3} \mathrm{O}_{4}$ & $\mathrm{H}_{2}+\mathrm{C}_{2} \mathrm{H}_{4}, 650^{\circ} \mathrm{C}$ & $\mathrm{H}_{2}+\mathrm{C}_{2} \mathrm{H}_{4}, 650^{\circ} \mathrm{C}$ & $\mathrm{Fe}-\mathrm{Fe}$ & N/A & $\mathrm{N} / \mathrm{A}$ & $\mathrm{N} / \mathrm{A}$ & N/A \\
\hline $\mathrm{Co}_{3} \mathrm{O}_{4}$ & $\mathrm{H}_{2}, 650^{\circ} \mathrm{C}$ & $\mathrm{H}_{2}, \mathrm{RT}$ & $\mathrm{Co}-\mathrm{Co}$ & 10.8 & 2.51 & 0.5 & 0.3 \\
\hline $\mathrm{Co}_{3} \mathrm{O}_{4}$ & $\mathrm{H}_{2}, 650^{\circ} \mathrm{C}$ & $\mathrm{H}_{2}, 650^{\circ} \mathrm{C}$ & $\mathrm{Co}-\mathrm{Co}$ & 10.8 & 2.49 & 13.0 & -1.6 \\
\hline $\mathrm{Co}_{3} \mathrm{O}_{4}$ & $\mathrm{H}_{2}+\mathrm{C}_{2} \mathrm{H}_{4}, 650^{\circ} \mathrm{C}$ & $\mathrm{H}_{2}+\mathrm{C}_{2} \mathrm{H}_{4}, 650^{\circ} \mathrm{C}$ & $\mathrm{Co}-\mathrm{Co}$ & 11.1 & 2.49 & 13.0 & -1.7 \\
\hline $\mathrm{FeCo}$ & $\mathrm{H}_{2}, 650^{\circ} \mathrm{C}$ & $\mathrm{H}_{2}, \mathrm{RT}$ & Fe-M & 6.9 & 2.48 & 0.5 & 3.5 \\
\hline $\mathrm{FeCo}$ & $\mathrm{H}_{2}, 650^{\circ} \mathrm{C}$ & $\mathrm{H}_{2}, 650^{\circ} \mathrm{C}$ & Fe-M & 6.9 & 2.45 & 11.0 & -1.3 \\
\hline $\mathrm{FeCo}$ & $\mathrm{H}_{2}+\mathrm{C}_{2} \mathrm{H}_{4}, 650^{\circ} \mathrm{C}$ & $\mathrm{H}_{2}, \mathrm{RT}$ & Fe-M & 7.4 & 2.48 & 0.0 & 2.8 \\
\hline $\mathrm{FeCo}$ & $\mathrm{H}_{2}+\mathrm{C}_{2} \mathrm{H}_{4}, 650^{\circ} \mathrm{C}$ & $\mathrm{H}_{2}+\mathrm{C}_{2} \mathrm{H}_{4}, 650^{\circ} \mathrm{C}$ & Fe-M & 7.4 & 2.46 & 11.0 & -0.8 \\
\hline $\mathrm{FeCo}$ & $\mathrm{H}_{2}, 650^{\circ} \mathrm{C}$ & $\mathrm{H}_{2}, \mathrm{RT}$ & Co-M & 7.2 & 2.48 & 0.5 & -2.8 \\
\hline $\mathrm{FeCo}$ & $\mathrm{H}_{2}, 650^{\circ} \mathrm{C}$ & $\mathrm{H}_{2}, 650^{\circ} \mathrm{C}$ & Co-M & 7.2 & 2.46 & 11.0 & -5.0 \\
\hline $\mathrm{FeCo}$ & $\mathrm{H}_{2}+\mathrm{C}_{2} \mathrm{H}_{4}, 650^{\circ} \mathrm{C}$ & $\mathrm{H}_{2}+\mathrm{C}_{2} \mathrm{H}_{4}, 650{ }^{\circ} \mathrm{C}$ & Co-M & 6.5 & 2.45 & 11.0 & -7.3 \\
\hline
\end{tabular}

Table 2 Fitted EXAFS data of the samples and references at the Co K-edge and Fe K-edge

While treatment with $\mathrm{H}_{2}+\mathrm{C}_{2} \mathrm{H}_{4}$ changed the size of the pure Fe nanoparticles, the same treatment had little effect on the XANES spectra at either edge of the reduced FeCo catalyst, indicating that the presence of Co in the alloy strongly modified the behaviour compared to the 
pure Fe nanoparticles (Figures. S.20 and S.21). There was also little change in the EXAFS for FeCo after treatment with $\mathrm{H}_{2}+\mathrm{C}_{2} \mathrm{H}_{4}$ (Fig. 3B) with the Fe-M $(\mathrm{M}=\mathrm{Fe}+\mathrm{Co})$ coordination number increasing very slightly from 6.9 to 7.4 while at the Co edge (fig. S.20) the Co-M coordination decreased slightly from 7.2 to 6.5 . The XANES and EXAFS results at $650{ }^{\circ} \mathrm{C}$ are similar to those at RT. From the data at both the Fe and the Co edges there was little change in the structure of the FeCo nanoparticles under reaction conditions, indicating a significant difference in behaviour compared to pure Fe.

(a)

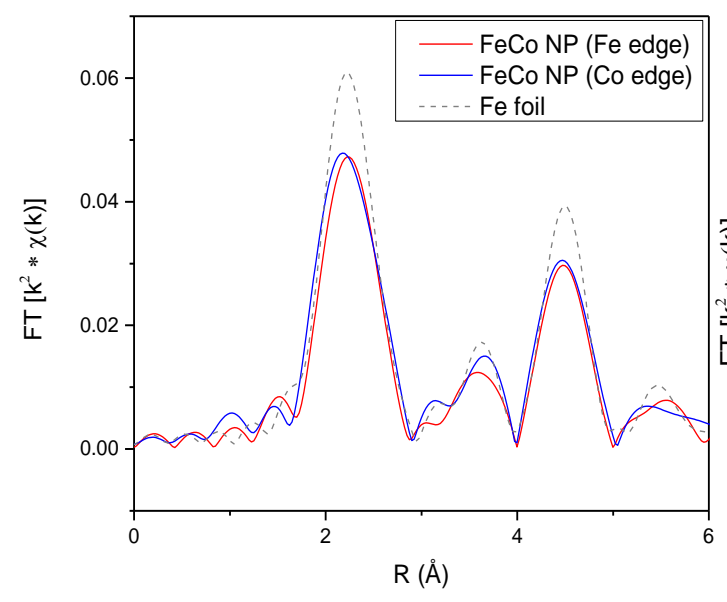

(b)

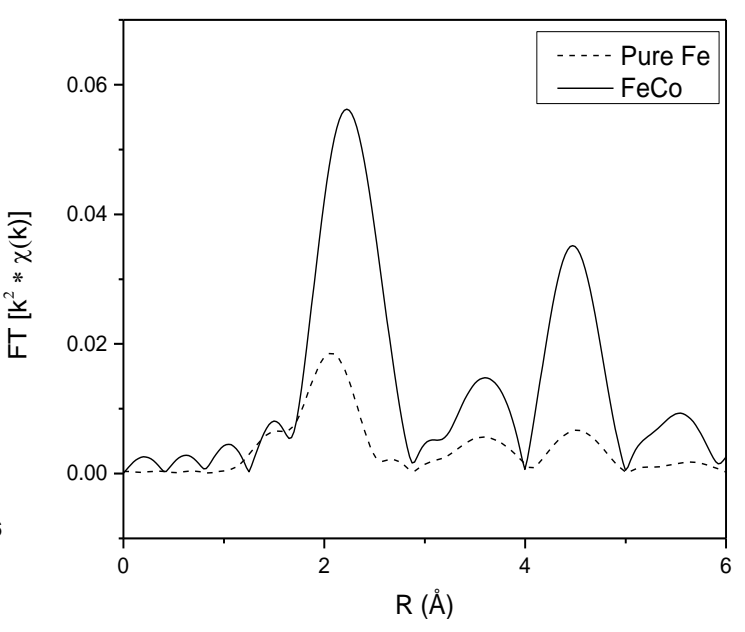

Figure 3 (A) Co and Fe K-edge magnitude of the FT of the EXAFS of FeCo nanoparticles in $\mathrm{H}_{2}$ showing the similarity in coordination at the Fe edge compared to the Co edge. Fe foil is shown for reference. (B) Fe K-edge magnitude of the FT of the EXAFS of FeCo and pure Fe after $\mathrm{H}_{2}+$ $\mathrm{C}_{2} \mathrm{H}_{4}$ treatment showing the large change in the EXAFS for pure Fe relative to FeCo. $\mathrm{k}^{2}: \Delta \mathrm{k}=$ $3.1-10.5 \AA^{-1}$. 
Figure 4A shows the evolution of the Raman spectra for $\mathrm{CoFe}_{2} \mathrm{O}_{4}$ when increasing the temperature during the ramp under $\mathrm{Ar} / \mathrm{H}_{2}$. At room temperature, the spectrum is characteristic of the one reported for $\mathrm{CoFe}_{2} \mathrm{O}_{4}$ with five peaks at 691, 615, 468, 307 and $205 \mathrm{~cm}^{-1}$ as expected for cubic $\mathrm{CoFe}_{2} \mathrm{O}_{4}$ from group symmetry. When the laser power is increased, an additional peak at $567 \mathrm{~cm}^{-1}$ is observed.[33, 34] From 25-200 ${ }^{\circ} \mathrm{C}$, Raman peaks down shift and broaden as expected. At around $325{ }^{\circ} \mathrm{C}, \mathrm{CoFe}_{2} \mathrm{O}_{4}$ is decomposed into another oxide displaying broad components at 662,540 and $300 \mathrm{~cm}^{-1}$, which are compatible with $\mathrm{Fe}_{3} \mathrm{O}_{4}$ [35] but not with $\alpha$ $\mathrm{Fe}_{2} \mathrm{O}_{3}, \gamma-\mathrm{Fe}_{2} \mathrm{O}_{3}$ or $\mathrm{FeO}$. An additional contribution at around $460 \mathrm{~cm}^{-1}$ can be attributed to residual $\mathrm{CoFe}_{2} \mathrm{O}_{4}$. No signature of $\mathrm{CoO}$ is observed. If $\mathrm{CoO}$ is indeed formed from the disproportionation of $\mathrm{CoFe}_{2} \mathrm{O}_{4}$ into $\mathrm{CoO}$ and $\mathrm{Fe}_{3} \mathrm{O}_{4}$ as reported in the literature,[36] it is possible that this oxide is reduced immediately to metallic Co (that cannot be identified by Raman spectroscopy) or that its crystallite size is very small. Increasing the temperature from 325 to 575 ${ }^{\circ} \mathrm{C}$ causes the signals to decrease in intensity and no Raman features of the catalyst oxide can be distinguished any more at $575^{\circ} \mathrm{C}$. We also noticed that if the catalyst was not sufficiently well calcined before the reducing treatment, $\mathrm{G}$ and $\mathrm{D}$ bands characteristic of carbonaceous deposits appear at $325{ }^{\circ} \mathrm{C}$ that are probably due to carbon contamination in the catalyst.[37] These deposits are unstable and disappear above $500^{\circ} \mathrm{C}$. After cooling to room temperature, a spectrum characteristic of $\mathrm{Fe}_{3} \mathrm{O}_{4}$ is clearly observed. From these results, the following scenario can be proposed. $\mathrm{CoFe}_{2} \mathrm{O}_{4}$ start decomposing into $\mathrm{CoO}$ and $\mathrm{Fe}_{3} \mathrm{O}_{4}$ between 200 and $325^{\circ} \mathrm{C} \mathrm{Fe}_{3} \mathrm{O}_{4}$ remains stable up to $575^{\circ} \mathrm{C}$ and is the only oxide phase observed after cooling down.[38]. It appears therefore that the active species that is responsible for the formation of CNFs is either $\mathrm{Fe}_{3} \mathrm{O}_{4}$ or $\mathrm{CoO}$. Experiments with pure $\mathrm{Fe}_{3} \mathrm{O}_{4}$ nanoparticles under the same conditions have proven that the former is the active species leading to the formation of CNFs. After catalyst 
reduction, we monitored the formation of FLG by introducing ethylene at $650{ }^{\circ} \mathrm{C}$ in the cell. As shown in Fig. 4B, characteristic bands due to FLG (G, D and G') start appearing within the first tens of seconds and saturate after 200-1000 s depending on the synthesis conditions. For the synthesis conditions tested (see section on Experimental), there was little modification of the spectral profile during growth; the G/D was close to 2 with a tendency to increase with time. The $\mathrm{G}^{\prime} / \mathrm{G}$ ratio was close to 0.5 and tended to increase with time.

A

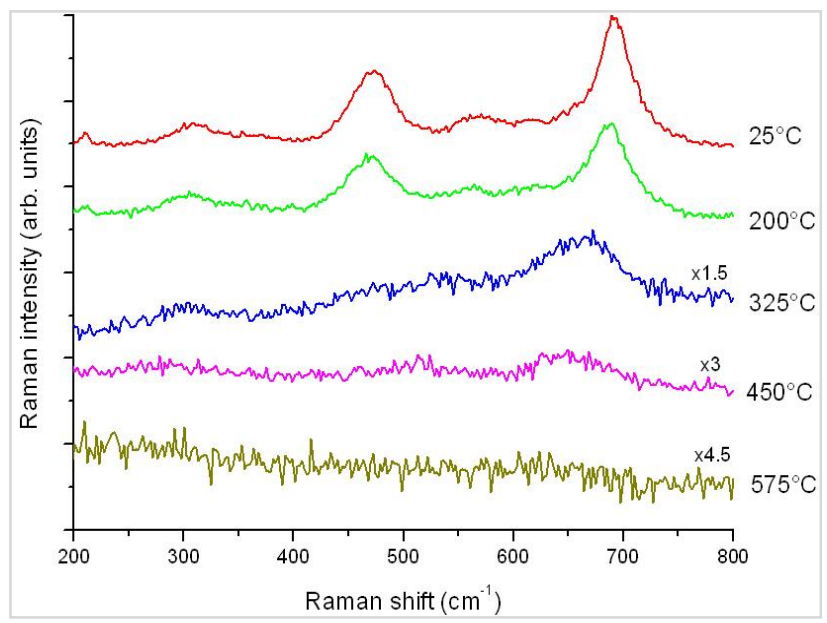

B

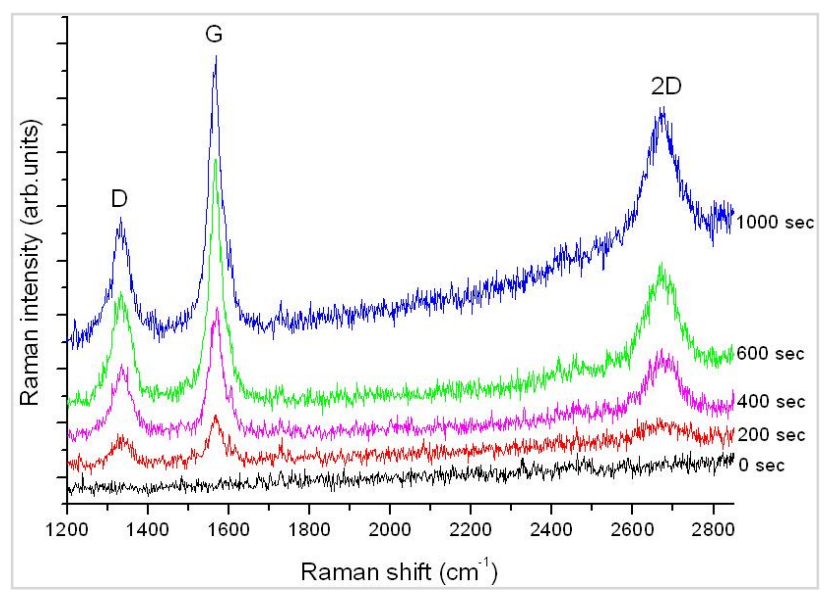


Figure 4 (A) Evolution of the Raman spectra of $\mathrm{CoFe}_{2} \mathrm{O}_{4}$ catalyst during ramping under $\mathrm{Ar} / \mathrm{H}_{2}$. (B) Raman spectra collected during FLG growth at $650{ }^{\circ} \mathrm{C}\left(\mathrm{C}_{2} \mathrm{H}_{4} 0.3 \mathrm{sccm} / \mathrm{H}_{2} 50 \mathrm{sccm} / \mathrm{Ar} 150\right.$ sccm). Laser wavelength $532 \mathrm{~nm}$. Acquisition time 20s.

Figure 5 shows the TEM images of the $\mathrm{CoFe}_{2} \mathrm{O}_{4}$ catalyst, at $0 \mathrm{~s}, 15 \mathrm{~s}, 2 \mathrm{~min}$ and $20 \mathrm{~min}$ of reaction in the presence of ethylene at $650{ }^{\circ} \mathrm{C}$. Figure $5 \mathrm{~A}$ shows a TEM image of the $10-14 \mathrm{~nm}$ $\mathrm{CoFe}_{2} \mathrm{O}_{4}$ nanoparticles used for FLG synthesis. Figure 5B shows the same particles after reduction for $1 \mathrm{~h}$ in $\mathrm{Ar} / \mathrm{H}_{2}$ atmosphere at $650{ }^{\circ} \mathrm{C}$ (body-centered cubic (bcc) FeCo alloy as determined from in situ EXAFS). XRD and EDX analysis confirmed that FeCo alloy with the $b c c$ structure (JCPDS file 01-087-0722) is present with an average crystallite size of $200 \mathrm{~nm}$. The composition of the alloy measured by EDX analysis gave a Co/Fe ratio of 33/66 for most of the crystals. After ethylene exposure for 15s, radially growing FLG flake (Fig. 5C) is seen spreading outward from the metal catalyst that appears to have suffered fragmentation (Fig. 5D). Also seen are very small nanoparticles $(2-3 \mathrm{~nm})$ dispersed on/in the graphene flake (inset Fig. 5D) arising from partial fragmentation of the original particles. The average sizes of these particles increases from 2 to $8 \mathrm{~nm}$ from $15 \mathrm{~s}$ to $20 \mathrm{~min}$ (Fig.5D-G) and the thickness of the graphene particles increased forming round flakes that curve at the edges. It appeared that the particles are not present on the graphene surface but buried in the interlayer spaces and hence their composition could not be determined. The 3D-TEM analysis of the sample exposed during 120 s to ethylene confirms this hypothesis (Fig. 5H). 


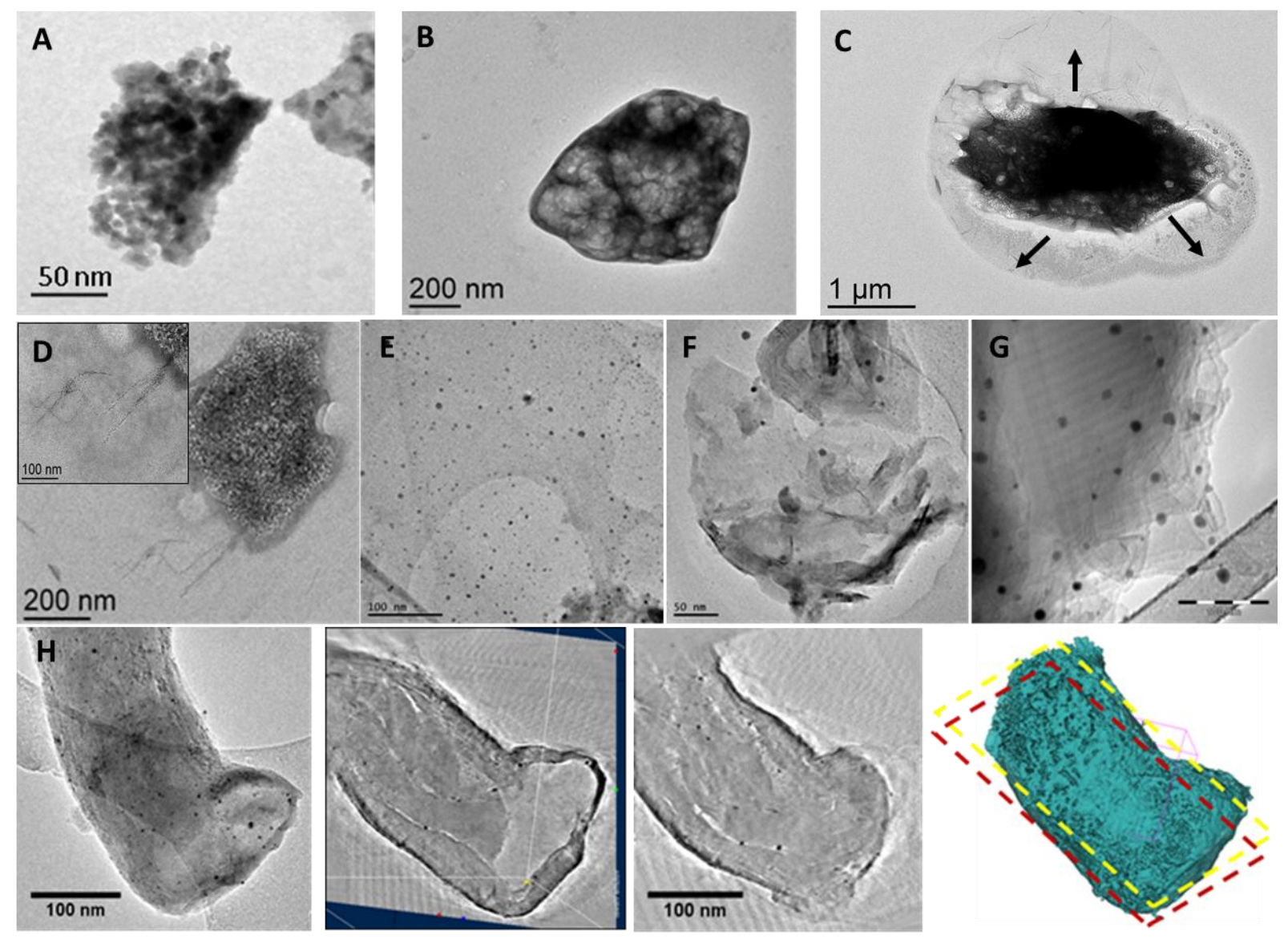

Figure 5 TEM images of (A) $\mathrm{CoFe}_{2} \mathrm{O}_{4}$ nanoparticles; (B) $\mathrm{CoFe}_{2} \mathrm{O}_{4}$ reduced in $\mathrm{H}_{2}+\mathrm{Ar}$ at $650{ }^{\circ} \mathrm{C}$; (C-D) after reaction with $\mathrm{C}_{2} \mathrm{H}_{4}$ at $15 \mathrm{~s}$; (E-F) 2min; and (G) 20 min.(H) Electron tomography of sample shown in Fig.5G: from left to right BF-TEM image at $0^{\circ}$ tilt from the tilt series; representative slices extracted from the reconstructed volume of the annealed aggregate; and 3D model of the external surface of the aggregate showing the position of the two chosen slices.

The slices extracted from the 3D reconstructed volume (Fig. 5H) highlight a tubular shape of the graphene within several well-defined channels inside. Moreover, the metallic nanoparticles are located preferentially in these channels and only very few of them are on the graphene surface. 
Note that the microstructure of the as-obtained FLG and the exact position of the nanoparticles with respect to the FLG surface are not visible from 2D-TEM observations. Hence the metal nanoparticles produced during the fragmentation of the FeCo alloy during carbon diffusion are located in nanometric channels. At the end of 2 min these particles appear to have coalesced to produce larger particles and at the same time, present in larger channels. At the end of $20 \mathrm{~min}$, these particles are present on the surface, from where they can be easily removed by acid washing. It is surprising that CNTs are not observed for these samples since previous studies have shown that $\mathrm{FeCo}$ metal particles in the 3-10 nm range size are excellent catalysts for the production of CNTs.[39] Supporting our catalysts on various substrates such as $\mathrm{Al}_{2} \mathrm{O}_{3}, \mathrm{MgO}$ or $\mathrm{SiO}_{2}$ led invariably to the selective formation of MWCNTs for all the supports tested.

The deposition of graphitic carbon accompanied by the fragmentation of the FeCo alloy catalyst leads us to suppose that a mechanism similar to the metal dusting process or a severe disruption of the substrate surface accompanied by metal carbon interdiffusion [17] could be operative in the early stages of the reaction. This is supported by the observation that increased iron in the catalyst leads to higher rates of dusting and $\mathrm{Co}_{3} \mathrm{O}_{4}$ shows a very low rate of carbon deposition (Figure 1E). The phenomenon of metal dusting has been intensively studied by metallurgists from wherein it is clear that the dusting mechanism is different depending on the nature of the active species. In Fe based alloys, formation of cementite followed by its decomposition is the usual pathway to metal dusting.[40] However, it has also been observed that even small additions of $\mathrm{Co}$ or Ni can prevent carbides from forming and dusting can take place by the direct dissolution of carbon in the metal matrix with the displaced metal particles moving towards the interphase with the gas phase, $[32,41]$ where they usually catalyse the formation of filamentous carbon.[42] In our samples, while intensive dusting has not been confirmed from EXAFS results, 
TEM results show that at least some of the Fe-Co alloy particles undergo dusting even though the fraction of such particles may be very limited. XRD of the samples at 9s, 2 min and $20 \mathrm{~min}$ show a reduction in crystallite sizes by $\sim 10 \%$ (Figure S.22). Hence, a mechanism involving direct dissolution of carbon may be operative in the present case, except that MWCNTs are not produced, presumably due to the slow diffusion of the particles towards the surface compared to the very rapid FLG deposition process (Fig. 1A). The selective formation of FLG indicates that its very rapid deposition hinders the metal particles from reaching the gas interphase. Instead, the small metal particles are found to coalesce and travel across the FLG layers by forming channels, presumably due to methanation reactions (Fig. 5H).[43] Since carbon diffusion occurs preferentially through grain boundaries, the dusting rate is related to the grain size of metal catalyst. Such behaviour has been observed in the past for growth of graphite over foils of platinum and nicket at $1000^{\circ} \mathrm{C}$. [19] In the present case, even though XRD of the reduced catalyst has yielded an average grain size of $200 \mathrm{~nm}$, HRTEM has shown that the grain sizes may be non-uniform with several grains on the particle edges with sizes in the 10-40 nm range, replicating the grain sizes of the starting oxide (Figure. S.23). Indeed, this observation may explain why only partial dusting is observed and large sized grains remain unaffected. Predictably, increase in cobalt content reduces the dusting rate, leading to lower rates of carbon deposition (Fig. 1E). Hence the FLG nucleation rate can be controlled by the composition of the catalyst. Thus, we believe that FLG growth is initiated on the large alloyed FeCo particles, and that dusting constitutes a side-reaction. More studies, including modelling using Density Functional Theory (DFT) are needed to confirm this. Finally, in situ Raman and TPR (Figure S.24) results confirm the disproportionation of the spinel at $450{ }^{\circ} \mathrm{C}$ to form the $\mathrm{FeCo}$ alloy at 
temperatures above $575^{\circ} \mathrm{C}$.[36] Hence, the active species in the formation of $\mathrm{CNFs}$ is $\mathrm{Fe}_{3} \mathrm{O}_{4}$, since dusting of $\mathrm{Fe}_{3} \mathrm{O}_{4}$ has been reported to take place in this temperature range.[40]

The results presented above demonstrate that oxide spinels are good catalysts for substrate free production of FLG. Even though the production yield is still low for very thin FLG, the flexibility of the process allows room for improvement. Detailed characterisation of FLG produced with spinel catalysts with different $\mathrm{A} / \mathrm{B}$ ratios needs to be carried out in order to control the graphene growth rate at the same time avoiding the formation of other forms of nanocarbon. The process has a strong potential for industrial applications since it uses low processing temperatures and greenhouse gases as carbon source. FLG with very low $(<4 \%)$ oxygen content can be produced and in situ doping with elements such as nitrogen or boron can be envisaged. First applications of our FLG samples, coated with Pd or metal free, have shown excellent catalytic activity for direct oxidation of alcohols [44] and aqueous sulphide oxidation reactions respectively.[45]

\section{Acknowledgements}

RB and PS thank P. Lonchambon for technical assistance, L. Datas, V. Collier and S. Leblond du Plouy (TEMSCAN, Toulouse University) for TEM and SEM images, E. Castillejos for XPS analysis and B. Machado for TPR measurements. This work has been carried out with the help of funding from the European Program POCO (Large scale collaborative project Grant agreement no.: CP-IP 213939-1) and ARKEMA FRANCE. X-ray adsorption spectroscopy studies by JTM and JRG were supported by the Institute for Atom-efficient Chemical Transformations (IACT), 
an Energy Frontier Research Center funded by the US Department of Energy, Office of Science,

and Office of Basic Energy Sciences. Use of the Advanced Photon Source is supported by the U.S. Department of Energy, Office of Science, and Office of Basic Energy Sciences, under Contract DE-AC02-06CH11357. MRCAT operations are supported by the Department of Energy and the MRCAT member institutions.

\section{References}

[1] Bonaccorso F, Lombardo A, Hasan T, Sun Z, Colombo L, Ferrari AC. Production and processing of graphene and 2d crystals. Materials Today. 2012;15(12):564-89.

[2] Van Noorden R. Production: Beyond sticky tape. Nature. 2012;483(7389):S32-S3.

[3] Lee S, Eom SH, Chung JS, Hur SH. Large-scale production of high-quality reduced graphene oxide. Chemical Engineering Journal. 2013;233(0):297-304.

[4] Geim AK, Novoselov KS. The rise of graphene. Nat Mater. 2007;6(3):183-91.

[5] Park S, Ruoff RS. Chemical methods for the production of graphenes. Nat Nano. 2009;4(4):217-24.

[6] Zimmermann S, Fatikow S. Nanorobotic handling of few-layer graphene membranes using a combined AFM/SEM/FIB setup. International Conference on Manipulation, Manufacturing and Measurement on the Nanoscale (3M-NANO), 2012 p. 102-7.

[7] Graphene, An Advanced Strategic High Tech Material. Global Research News. 2013(July 06 ).

[8] Zhang Y, Zhang L, Zhou C. Review of Chemical Vapor Deposition of Graphene and Related Applications. Accounts of Chemical Research. 2013;46(10):2329-39.

[9] Reina A, Jia X, Ho J, Nezich D, Son H, Bulovic V, et al. Large Area, Few-Layer Graphene Films on Arbitrary Substrates by Chemical Vapor Deposition. Nano Letters. 2008;9(1):30-5.

[10] Bae S, Kim H, Lee Y, Xu X, Park J-S, Zheng Y, et al. Roll-to-roll production of 30-inch graphene films for transparent electrodes. Nat Nano. 2010;5(8):574-8.

[11] Chang Seok L, Costel Sorin C, Waleed M, Bérengère L, Marc C, Marc C, et al. Synthesis of conducting transparent few-layer graphene directly on glass at $450^{\circ} \mathrm{C}$. Nanotechnology. 2012;23(26):265603.

[12] Li Z, Zhu H, Xie D, Wang K, Cao A, Wei J, et al. Flame synthesis of few-layered graphene/graphite films. Chemical Communications. 2011;47(12):3520-2.

[13] Wang X, You H, Liu F, Li M, Wan L, Li S, et al. Large-Scale Synthesis of Few-Layered Graphene using CVD. Chemical Vapor Deposition. 2009;15(1-3):53-6.

[14] Bartholomew CH. Carbon Deposition in Steam Reforming and Methanation. Catalysis Reviews. 1982;24(1):67-112.

[15] Hofer LJE, Sterling E, McCartney JT. Structure of Carbon Deposited from Carbon Monoxide on Iron, Cobalt and Nickel. The Journal of Physical Chemistry. 1955;59(11):1153-5.

[16] Robertson SD. Carbon formation from methane pyrolysis over some transition metal surfaces-I. Nature and properties of the carbons formed. Carbon. 1970;8(3):365-74. 
[17] Robertson SD. Carbon formation from methane pyrolysis over some transition metal surfaces-II. Manner of carbon and graphite formation. Carbon. 1972;10(2):221-9.

[18] Baker RTK, Feates FS, Harris PS. Continuous electron microscopic observation of carbonaceous deposits formed on graphite and silica surfaces. Carbon. 1972;10(1):93-6.

[19] Presland AEB, Walker Jr PL. Growth of single-crystal graphite by pyrolysis of acetylene over metals. Carbon. 1969;7(1):1-8.

[20] Walker PL, Rakszawski JF, Imperial GR. Carbon Mixture over Iron Catalysts. II. Rates of Carbon Formation. The Journal of Physical Chemistry. 1959;63(2):140-9.

[21] Walker PL, Rakszawski JF, Imperial GR. Carbon Formation from Carbon MonoxideHydrogen Mixtures over Iron Catalysts.I. Properties of Carbon Formed. The Journal of Physical Chemistry. 1959;63(2):133-40.

[22] Bacsa R, Serp P, inventors; Graphene production method and graphene obtained by said method. 2013 WO2013093350 A1.

[23] Philippe R, Caussat B, Falqui A, Kihn Y, Kalck P, Bordère S, et al. An original growth mode of MWCNTs on alumina supported iron catalysts. Journal of Catalysis. 2009;263(2):34558.

[24] Beausoleil J, Bacsa R., Caussat, B. , Serp P. ,, inventor Method for producing an assembly of carbon nanotubes and graphene. 2013 WO 2013093358 A1 2013.

[25] Ümit Ö, Yahya A, Hadis M. Microwave ferrites, part 1: fundamental properties. Journal of Materials Science: Materials in Electronics. 2009.

[26] Pimenta MA, Dresselhaus G, Dresselhaus MS, Cancado LG, Jorio A, Saito R. Studying disorder in graphite-based systems by Raman spectroscopy. Phys Chem Chem Phys. 2007;9(11):1276-91.

[27] Campos-Delgado J, Botello-Méndez AR, Algara-Siller G, Hackens B, Pardoen T, Kaiser $\mathrm{U}$, et al. CVD synthesis of mono- and few-layer graphene using alcohols at low hydrogen concentration and atmospheric pressure. Chemical Physics Letters. 2013;584(0):142-6.

[28] Ferrari AC, Meyer JC, Scardaci V, Casiraghi C, Lazzeri M, Mauri F, et al. Raman Spectrum of Graphene and Graphene Layers. Physical Review Letters. 2006;97(18):187401.

[29] Cançado LGT, K. Enoki, T. Endo, M.-Kim, Y.A.-Mizusaki, H. Speziali, N.L.Jorio, A. Pimenta, M.A. Measuring the degree of stacking order in graphite by Raman spectroscopy. Carbon. 2008;46(2):3.

[30] Cançado LG, Takai K, Enoki T, Endo M, Kim YA, Mizusaki H, et al. General equation for the determination of the crystallite size La of nanographite by Raman spectroscopy. Applied Physics Letters. 2006;88(16):163106.1-3.

[31] Tuinstra F, Koenig JL. Raman Spectrum of Graphite. The Journal of Chemical Physics. 1970;53(3):1126-30.

[32] Chun CM, Mumford JD, Ramanarayanan TA. Mechanisms of Metal Dusting Corrosion of Iron. Journal of The Electrochemical Society. 2002;149(7):B348-B55.

[33] Da Silva SW, Melo TFO, Soler MAG, Lima ECD, Da Silva MF, Morais PC. Stability of citrate-coated magnetite and cobalt-ferrite nanoparticles under laser irradiation: a Raman spectroscopy investigation. Magnetics, IEEE Transactions on. 2003;39(5):2645-7.

[34] Wang Z, Downs RT, Pischedda V, Shetty R, Saxena SK, Zha CS, et al. High-pressure xray diffraction and Raman spectroscopic studies of the tetragonal spinel. Physical Review B. 2003;68(9):094101.

[35] De Faria D, Venâncio Silva S, De Oliveira M. Raman microspectroscopy of some iron oxides and oxyhydroxides. J Raman Spectrosc. 1997;28(11):873-8. 
[36] Nilsen MH, Nordhei C, Ramstad AL, Nicholson DG, Poliakoff M, Cabañas A. XAS (XANES and EXAFS) Investigations of Nanoparticulate Ferrites Synthesized Continuously in Near Critical and Supercritical Water. The Journal of Physical Chemistry C. 2007;111(17):625262.

[37] Chourpa I, Douziech-Eyrolles L, Ngaboni-Okassa L, Fouquenet J-F, Cohen-Jonathan S, Souce M, et al. Molecular composition of iron oxide nanoparticles, precursors for magnetic drug targeting, as characterized by confocal Raman microspectroscopy. Analyst. 2005;130(10):1395403.

[38] Venugopal A, Scurrell MS. Low temperature reductive pretreatment of $\mathrm{Au} / \mathrm{Fe} 2 \mathrm{O} 3$ catalysts, TPR/TPO studies and behaviour in the water-gas shift reaction. Applied Catalysis A: General. 2004;258(2):241-9.

[39] Reshetenko TV, Avdeeva LB, Ushakov VA, Moroz EM, Shmakov AN, Kriventsov VV, et al. Coprecipitated iron-containing catalysts (Fe-Al2O3, Fe-Co-Al2O3, Fe-Ni-Al2O3) for methane decomposition at moderate temperatures: Part II. Evolution of the catalysts in reaction. Applied Catalysis A: General. 2004;270(1-2):87-99.

[40] Szakalos P. Mechanisms of Metal Dusting Thesis dissertaton, Royal Institute of Technology, Stockholm, Sweden 2004.

[41] Chun CM, Mumford JD, Ramanarayanan TA. Metal Dusting Corrosion of Cobalt. Journal of The Electrochemical Society. 2003;150(2):B76-B82.

[42] Young DJ, Zhang J, Geers C, Schütze M. Recent advances in understanding metal dusting: A review. Materials and Corrosion. 2011;62(1):7-28.

[43] Kustov AL, Frey AM, Larsen KE, Johannessen T, Nørskov JK, Christensen CH. CO methanation over supported bimetallic $\mathrm{Ni}-\mathrm{Fe}$ catalysts: From computational studies towards catalyst optimization. Applied Catalysis A: General. 2007;320(0):98-104.

[44] Machado BF, Marchionni A, Bacsa RR, Bellini M, Beausoleil J, Oberhauser W, et al. Synergistic effect between few layer graphene and carbon nanotube supports for palladium catalyzing electrochemical oxidation of alcohols. Journal of Energy Chemistry. 2013;22(2):296304.

[45] Lemos BRS, Teixeira IF, Machado BF, Alves MRA, de Mesquita JP, Ribeiro RR, et al. Oxidized few layer graphene and graphite as metal-free catalysts for aqueous sulfide oxidation. Journal of Materials Chemistry A. 2013;1(33):9491-7. 